\title{
Pseudomyxoma peritonei of a mature ovarian teratoma caused by mismatch repair deficiency in a patient with Lynch syndrome: a case report
}

Yoshimasa Gohda ${ }^{1}$, Rei Noguchi², Tomoko Horie ${ }^{1}$, Toru Igari ${ }^{3}$, Harumi Nakamura ${ }^{3}$, Yasunori Ohta ${ }^{4}$, Kiyoshi Yamaguchi ${ }^{2}$, Tsuneo Ikenoue ${ }^{2}$, Seira Hatakeyama ${ }^{2}$, Nozomi Yusa ${ }^{5}$, Yoichi Furukawa ${ }^{2,5,6^{*}}$ and Hideaki Yano ${ }^{1}$

\begin{abstract}
Background: Pseudomyxoma peritonei (PMP) is a rare disease with an estimated incidence of 1-2 cases per million individuals per year. PMP is characterized by the accumulation of abundant mucinous or gelatinous fluid derived from disseminated tumorous cells. Most of the tumorous cells are originated from rupture of appendiceal neoplasms, but some are from the metastasis of cancer of the colon, ovary, fallopian tube, urachus, colorectum, gallbladder, stomach, pancreas, lung and breast. Although frequent mutations in KRAS and/or GNAS genes have been reported, precise molecular mechanism underlying PMP remains to be elucidated. It is of note that mucinous tumour is one of the frequent histological features of colorectal cancer (CRC) in Lynch syndrome (LS), an autosomal dominantly inherited disease caused by a germline mutation of the DNA mismatch repair (MMR) genes including human mutL homolog 1 (MLH1), human mutS homolog 2 (MSH2), human mutS homolog 6 (MSH6), and postmeiotic segregation increased 2 (PMS2). Therefore, typical LS-associated tumours show mismatch repair instability. Although LS patients are most strongly predisposed to CRC, PMPs from mucinous CRC have not been reported in LS patients.
\end{abstract}

Case presentation: In this report, we report a case of PMP originating from an ovarian teratoma in a LS patient. The patient had surgical treatment of PMP arising from an ovarian teratoma at the age of 38 years, and later developed a transverse colon cancer at the age of 40. The patient's family history fulfilled the Amsterdam criteria, and genetic analysis of the peripheral leukocytes identified a germ line mutation in the MLH1 gene (MLH1 c.1546dupC p.Q516PfsX3). Interestingly, immunohistochemical staining showed that the expression of $\mathrm{MLH1}$ was lost in the colon cancer as well as the ovarian teratoma. Consistent with the loss of MLH1 expression, both tumours showed high microsatellite instability (MSI-H).

Conclusion: This case suggested that LS patients may develop various types of tumours including ovarian PMP, and that mismatch repair deficiency may play a role in the development of PMP derived from, at least, a part of ovarian teratomas.

Keywords: Pseudomyxoma peritonei, Ovarian teratoma, Lynch syndrome, Mismatch repair, Microsatellite instability

\footnotetext{
* Correspondence: furukawa@ims.u-tokyo.ac.jp

${ }^{2}$ Division of Clinical Genome Research, Institute of Medical Science, The University of Tokyo, 4-6-1 Shirokanedai, Minato-ku, 108-8639 Tokyo, Japan ${ }^{5}$ Department of Applied Genomics, Research Hospital, Institute of Medical Science, The University of Tokyo, 4-6-1 Shirokanedai, Minato-ku, 108-8639 Tokyo, Japan

Full list of author information is available at the end of the article
} 


\section{Background}

Pseudomyxoma peritonei (PMP) is a very rare disorder, and a nationwide study in Netherland disclosed that the estimated incidence of PMP is 1-2 cases per million individuals per year [1]. The condition is characterized by the accumulation of abundant mucinous or gelatinous fluid that is produced from tumorous cells disseminated in the abdominal cavity and pelvis [2]. Patients with PMP usually suffer from abdominal distension, change of body weight, abdominal or pelvic pain, and/or digestive disorder by the accumulation of ascites and/or the progression of disseminated lesion. Tumour cells of PMP are most frequently derived from rupture of appendiceal neoplasms, but occasionally from the tumours in other organs including the ovary [1, 3], fallopian tube [4], urachus $[1,5]$, colorectum $[1,6]$, gallbladder [7], stomach [8], pancreas [1,9], lung [10] and breast [11]. The average onset of the disease is reportedly from 49 to 62 years old, which is earlier than other abdominal malignancies [1, 12]. Several molecular studies have disclosed that frequent mutations in KRAS and GNAS are involved in appendiceal PMP [13-18]. However precise molecular mechanism of PMP remains to be elucidated.

Regarding the treatment of PMP, cytoreductive surgery (CRS) combined with hyperthermic intraperitoneal chemotherapy (HIPEC) has greatly improved the prognosis of patients. A recent study of 1000 appendiceal PMPs revealed that 738 patients treated with complete CRS and HIPEC showed $87.4 \%$ of 5 -year and $70.3 \%$ of 10-year survival [19]. However, little is known about the prognosis of PMP originated from ovarian teratoma.

Lynch syndrome (LS) or hereditary non-polyposis colorectal cancer (HNPCC) is an autosomal dominantly inherited syndrome accounting for $2-7 \%$ of all colorectal cancers (CRC) [20, 21]. It is caused by a germline mutation of the DNA mismatch repair genes (MMR) including human mutL homolog 1 (MLH1), human mutS homolog 2 (MSH2), and to a lesser extent, human mutS homolog 6
(MSH6), and postmeiotic segregation increased 2 (PMS2). LS is characterized by the susceptibilities for various LSassociated tumours such as colorectal, endometrial, stomach, small bowel, ovarian, urothelial, bladder, and biliary tract cancers [22, 23]. Ovarian cancer is one of Lynch syndrome-related extra-colorectal neoplasms and the life time risks are $4-12 \%$. The mean age at diagnosis of the ovarian cancers is 42.5 years and approximately $30 \%$ of the cancers is diagnosed before the age of 40 years [24]. The pathology type of Lynch syndrome-related ovarian cancers is similar to sporadic ovarian cancers including mostly epithelial cell-derived tumours. Even in sporadic ovarian cancers, 3-8\% of ovarian mucinous tumours occur from mature cystic teratoma which indicates that teratoma is a very rare form of ovarian tumours [25]. Although the frequency of mucinous colorectal cancer and that of ovarian tumours are higher in the LS patients compared with normal population, PMP has never been reported in LS patients. Most patients with Lynch syndrome are clinically identified by the revised Amsterdam's criteria, or the revised Bethesda's guideline for the test of microsatellite instability. The tumours associated with LS patients commonly show microsatellite instability, a hallmark of mismatch repair deficiency. In addition to testing for microsatellite instability, immunohistochemical staining of responsible gene products is often used for the screening of the deficiency.

Here, we report for the first time, a case of ovarian PMP in a patient with Lynch syndrome, and show the association of the ovarian tumour with mismatch repair deficiency.

\section{Case presentation}

A 38 year-old woman presented with progressive abdominal distension. Contrasted abdomino-pelvic CT revealed that the distended abdominal cavity of the patient was filled with low-density fluid, and showed a degree of scalloping sign on the liver surface (Fig. 1a). A 7-cm sized multi-

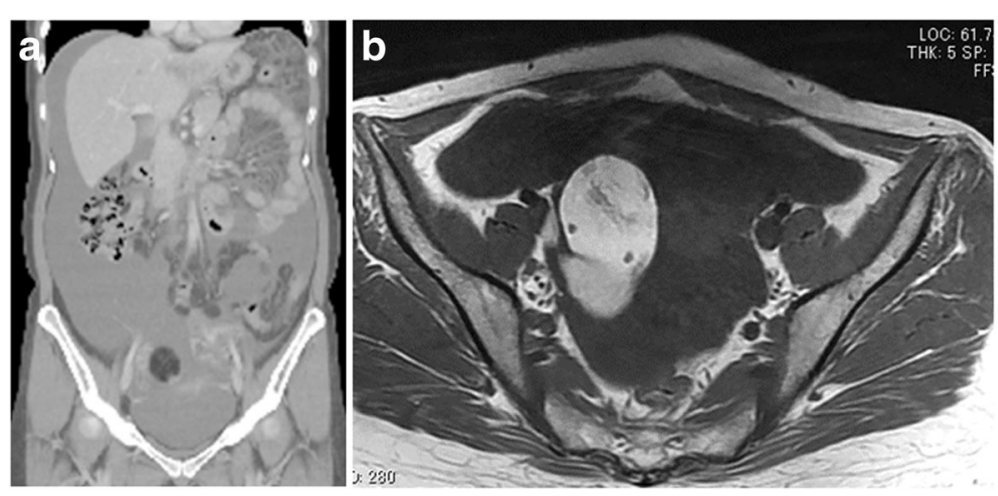

Fig. 1 a Contrasted computed tomography showed distended abdominal cavity filled with low-density fluid. b A pelvic magnetic resonance image of the right ovarian mass with high signal intensity on T2-weighted image 
locular cystic mass containing fat tissue, which was thought to be originating from the right ovary, was identified in the pelvis. However, the patient's appendix and left ovary appeared normal. Pelvic magnetic resonance image showed cystic component with high signal intensity on T1- and T2weighted images, and fat-saturated T1-weighted images showed a drop in the signal intensity of the right ovarian mass (Fig. 1b). These imaging studies suggested a teratoma of the right ovary resulting in pseudomyxoma peritonei. Colonoscopy and gastroscopy were normal. The laboratory test of tumour markers showed that CEA, CA19-9, and CA125 were elevated to $15.5 \mathrm{ng} / \mathrm{ml}$ (Upper normal limit $[\mathrm{UNL}]<5 \mathrm{ng} / \mathrm{ml}), 59.8 \mathrm{U} / \mathrm{ml}(\mathrm{UNL}<37 \mathrm{U} / \mathrm{ml})$, and $39.7 \mathrm{U} / \mathrm{ml}(\mathrm{UNL}<35 \mathrm{U} / \mathrm{ml})$, respectively.

Importantly, the proband's family history completely fulfilled the Amsterdam criteria (Fig. 2a). The mother suffered from appendiceal cancer, ascending colon cancer, and endometrial cancer, a maternal brother died of rectal cancer at the age of 51, and an older sister of the patient died of sigmoid colon cancer at the age of 31, suggesting the possible diagnosis of LS. After genetic counselling, a written informed consent was obtained from the patient, and a genetic test was performed using DNA from the patient's peripheral blood. As a result, a germ line mutation consisting of a single nucleotide duplication of C (c.1546dupC, p.Q516PfsX3) in codon516 was identified in the $M L H 1$ gene (Fig. 2b). This mutation was judged as a deleterious mutation because the duplication resulted in a frameshift mutation inducing a truncation of the MLH1 protein.

An open laparotomy was performed, where abundant mucinous ascites mixed with hair was found in the peritoneal cavity (Fig. 3a). In addition, surgical exploration identified a ruptured left ovarian cystic mass of $16 \mathrm{~cm}$ in size and a non-ruptured right ovarian cystic mass of $7.5 \mathrm{~cm}$, but the appendix was normal. The patient underwent complete cytoreductive surgery (CRS) including total abdominal hysterectomy with bilateral salpingo-oophorectomy, total peritonectomy, appendicectomy, greater and lesser omentectomy, splenectomy, cholecystectomy, and resection of the liver capsule. Following the CRS, hyperthermic intraperitoneal chemotherapy (HIPEC) was added with mitomycin $\mathrm{C}$ at $10 \mathrm{mg} / \mathrm{m}^{2}$ for $1 \mathrm{~h}$. The post-operative course was uneventful.

Both ovarian tumours were filled with copious gelatinous fluids containing multiple hairs and yellowish sebaceous materials (Fig. 3b). Histological examination revealed that the right ovarian tumour was a mature cystic teratoma accompanied by an endometriotic cyst. It was reported that 3 to $8 \%$ of primary ovarian mucinous tumours are accompanied by teratoma [26-28]. The teratoma-associated mucinous tumours were also described to show various histological appearances such as cystadenomatous, borderline tumour-like to adenocarcinoma, tumours with entity of goblet cell carcinoid, and low grade adenomatous mucinous tumours. Consistently, the left tumour was a mixture of mucinous cystic tumour and teratomatous lesion. Most of the tumour cells showed mild cellular atypia but complex structural changes classifying the tumour into an intermediate malignancy (Fig. 3c). Additionally, a part of the tumour demonstrated stromal invasion, which corresponds to moderately differentiated adenocarcinoma. The teratomatous tumours predominantly consist of ectodermal components such as skin and its adnexa, cartilage, tracheobronchial epithelium, and included mucin-producing epithelium depicting lower intestinal tract (Fig. 3d). Immunohistochemical study revealed that the left ovarian tumour with intermediate malignancy was negative for CK7 (Fig. 3e), and diffusely positive for CK20 and CDX2 suggesting that it has a lower intestinal phenotype (Fig. 3f and g). However, the region of moderately differentiated

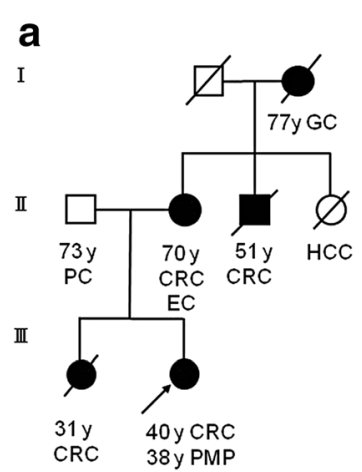

b

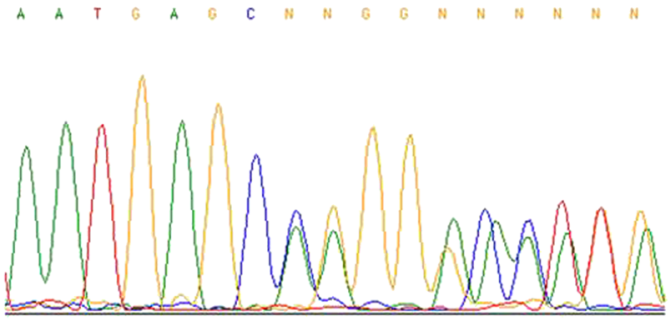

wild type $A$ A $T$ G $A$ G $C$ A $A$ G G G A

mutant $A$ A T G A G

Fig. 2 a The family tree of the patient. The patient (proband) is indicated by an arrow. Males and females are illustrated by squares and circles, respectively. Unaffected and affected individuals are indicated by open and closed symbols, respectively. Persons deceased are shown by a slash on the symbol. Histories of malignancy and the age of diagnosis are described under the symbols. CRC, colorectal cancer; PC, pancreatic cancer; EC, endometrial cancer; GC, gastric cancer; HCC, hepatocellular carcinoma. b Sequence chromatogram containing the mutation (MLH1 c.1546dupC, p.Q516PfsX3). Codon 516 is underlined 

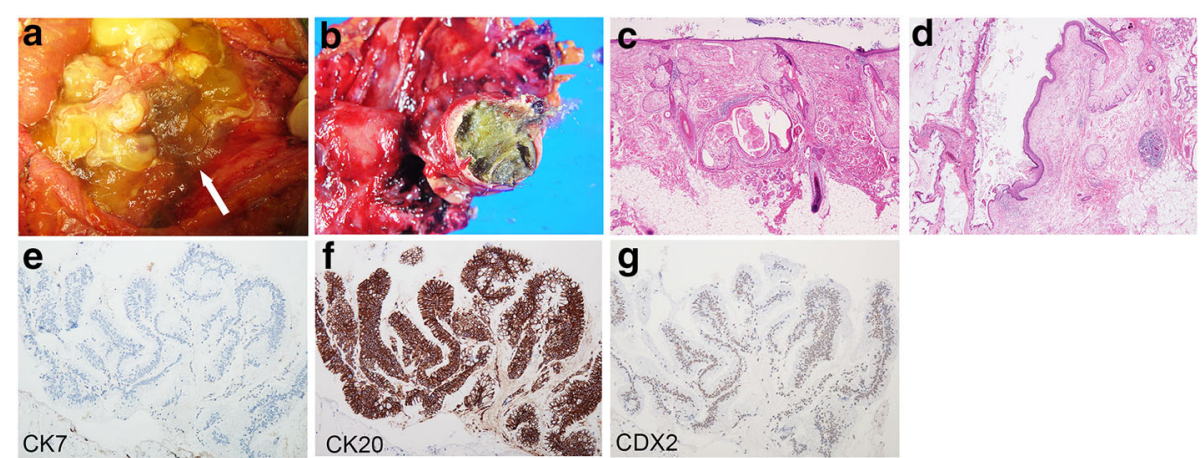

K20

$\mathrm{CDX} 2$

Fig. 3 a A picture of the patient's abdominal cavity. Arrow indicates a hair in the abdominal cavity. b Macroscopic appearance of the patient's ovary. c Borderline lesion containing epithelium with severe atypia in the teratoma. $\mathbf{d}$ Histology of the right teratoma containing various differentiations such as squamous epithelium, hair follicles, sebaceous materials, and mucinous epithelium. e, $\mathbf{f}$, and $\mathbf{g}$ Immunohistochemical staining of the mucinous epithelium with anti-CK7 (e), anti-CK20 (f), and anti-CDX2 (g) antibodies

adenocarcinoma was positive for CK7 and CDX2, and negative for CK20, indicating aberrant cytokeratin staining pattern due to malignant transformation. Histological examination of the peritoneum, omentum and implants found mucinous material and a small amount of tumour cells (pseudomyxoma). It is of note the histology of the appendix was normal. These data suggested that the pseudomyxoma peritonei may result from mucinproducing epithelial cells derived from ovarian teratoma.

To clarify the aetiology of the ovarian tumour, we carried out microsatellite instability (MSI) analysis with the Bethesda panel (D2S123, D5S346, D17S250, BAT25, and BAT26). Interestingly, the DNA from the left ovarian tumour was positive for MSI in three of the five markers (Fig. 4a) indicating high microsatellite instability (MSI-high). Additional immunohistochemical analysis showed positive staining for $\mathrm{MSH} 2$ and negative for MLH1 and PMS2 (Fig. 4b-d), which is in complete agreement with the germ line mutation in $M L H 1$. These data suggested that the deficiency of mismatch repair system was associated with the ovarian tumour.

After the surgery for PMP, the patient was enrolled in a surveillance programme of PMP. In the 2-yearly colonoscopy, a transverse colon cancer of $2.5 \mathrm{~cm}$ in size was identified. The patient underwent right hemicolectomy where no recurrence of PMP was detected. Histological examination of the tumour revealed tubular adenocarcinoma of the colon with an UICC stage of pT2N0M0 (data not shown).

Since our case underwent complete CRS and HIPEC for PMP and curative operation for transverse colon cancer, a good prognosis of the patient is expected. However, if recurrent disease may appear in the patient, we may need to

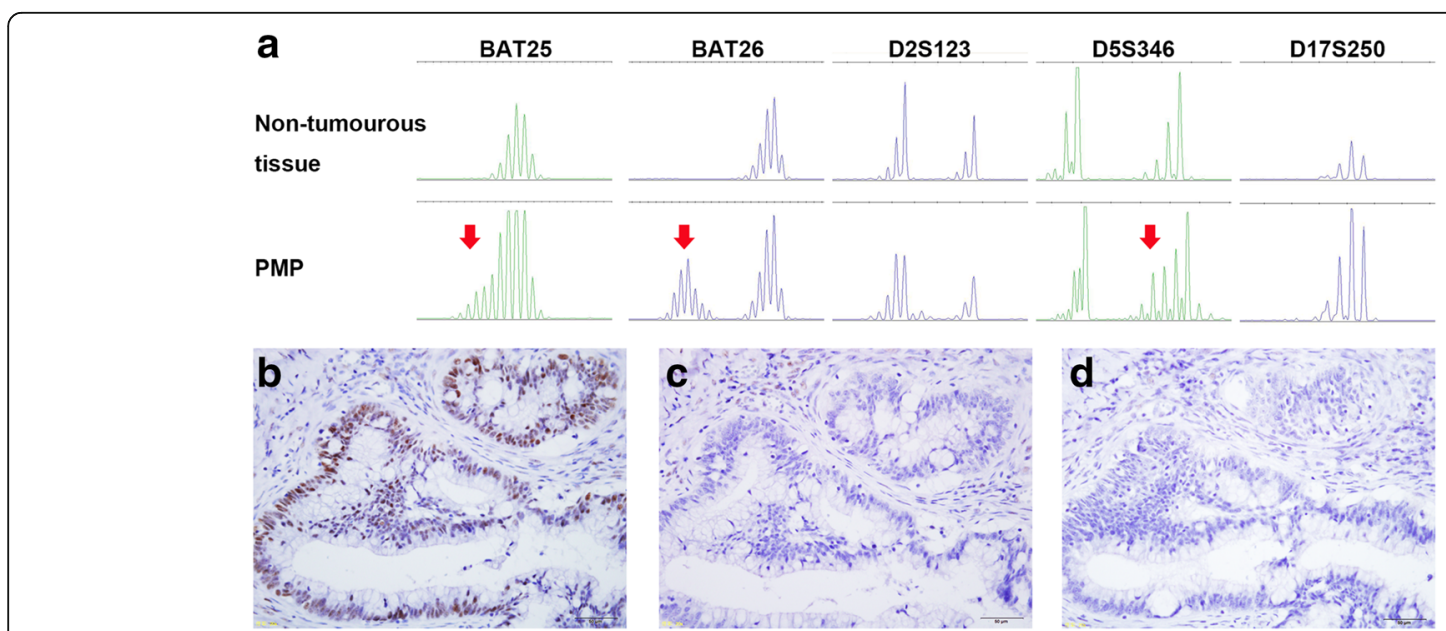

Fig. 4 a Microsatellite analysis of the PMP. Arrows depict aberrant peaks in tumorous tissues compared with non-tumorous tissues indicating microsatellite instability-high. b-d Immunohistochemical staining of mismatch repair proteins using anti-MSH2 (b), anti-MLH1 (c), and anti-PMS2 (d) antibodies 
consider immune checkpoint inhibitors for the treatment, because mismatch repair-deficient tumours are more effective to anti-programmed death 1 (PD1) monoclonal antibodies [29].

\section{Conclusion}

So far three groups have reported six PMPs derived from ovarian mature cystic teratoma $[3,25,30]$. In the papers, data of immunohistochemical staining are shown for six tumours. One of the six was partly $\mathrm{CK} 7+$, and diffuse $\mathrm{CK} 20+$ and diffuse CDX2+ [25], the second case was partly $\mathrm{CK} 7+$, and diffuse CK20+ and partly CDX2+ [25], the third to fifth cases were all CK7- and diffuse CK20+ [3], and the sixth case was CK7+, CK20+, and CDX2+ [30]. These data may imply that both lower-gastrointestinal tract type mucinous tumours and primary ovarian mucinous tumours developed in the ovarian teratoma may become the origin of PMPs. In this case, the patient's teratoma contained various types of epithelium, and the majority of cystic lesion was shown to be lined with mucinous epithelium similar to lower intestinal tract by histological and immunological examination (CK7-/CK20+/CDX2+). In addition, the epithelial cells showed from benign to malignant transformation. The region of moderately differentiated adenocarcinoma in the cystic teratoma with the positive immunohistochemical staining for CK7, strongly suggested that the PMP should have originated from the primary ovarian mucinous epithelium in the teratoma.

This is the first report of PMP originating from an ovarian mature teratoma in a Lynch syndrome patient. Two reports of cystic teratoma in Lynch syndrome have been archived in public databases. One of the two is a case in a Taiwanese LS family [30], and the other is a case of Muir-Torre syndrome, a subtype of Lynch syndrome involving a combination of sebaceous neoplasms of the skin and internal malignancies [31]. However, these two teratoma cases did not accompany PMP. Importantly, the latter case showed loss of MSH2 expression in the sebaceous adenoma within mature cystic ovarian teratoma suggesting the involvement of mismatch repair deficiency. Although the genetic changes associated with teratogenesis have not been clarified, impaired mismatch repair machinery might result in the genetic changes associated with the teratomas.

In conclusion, mismatch repair defect may cause PMP through the development of ovarian tumour, one of the extra-colorectal tumours associated with Lynch syndrome. Further studies will clarify the molecular mechanisms underlying PMP originating from ovarian teratoma.

\section{Abbreviations}

CRC: Colorectal cancer; CRS: Cytoreductive surgery; HIPEC: Hyperthermic intraperitoneal chemotherapy; HNPCC: Hereditary non-polyposis colorectal cancer; LS: Lynch syndrome; MLH1: Human mutL homolog 1.

MMR: Mismatch repair; MSH2: Human mutS homolog 2; MSH6: Human mutS homolog 6; MSI: Microsatellite instability; PD1: Programmed death 1; PMP: Pseudomyxoma peritonei; PMS2: Postmeiotic segregation increased 2

\section{Acknowledgement}

This work was supported in part by the Grant-in-Aid (\#25280105) from the Japan Society for the Promotion of Science.

Funding

This work was supported in part by the Japan Society for the Promotion of Science [grant number: the Grant-in-Aid (\#16H01569)].

\section{Availability of data and material}

The datasets during and/or analysed during the current study is available from the corresponding author upon reasonable request.

\section{Authors' contributions}

TIkenoue, YF and HY designed the report. RN, KY, SH, and NY performed the genetic analysis. YG, TH, and HY collected the patient's clinical data. TIgari, $\mathrm{HN}$ and $\mathrm{YO}$ evaluated the patient's pathological data. YG and RN analysed the data. YG, RN, YF and HY wrote the paper. All authors read and approved the final manuscript.

\section{Competing interests}

The authors declare that they have no competing interests.

\section{Consent for publication}

An additional informed consent was obtained from the patient to the publication of this case report and any accompanying images.

\section{Ethics approval and consent to participate}

This study was approved by the institutional review boards of the Institute of Medical Science, The University of Tokyo (IMSUT-IRB \#26-67-1118), and National Center for Global Health and Medicine. A written informed consent was obtained from the patient to the participation in this study.

\section{Author details}

${ }^{1}$ Division of Colorectal Surgery, Department of Surgery, National Center for Global Health and Medicine, 1-21-1 Toyama, Shinjuku-ku, 162-8655 Tokyo, Japan. ${ }^{2}$ Division of Clinical Genome Research, Institute of Medical Science, The University of Tokyo, 4-6-1 Shirokanedai, Minato-ku, 108-8639 Tokyo, Japan. ${ }^{3}$ Pathology Division of Clinical Laboratory, National Center for Global Health and Medicine, 1-21-1 Toyama, Shinjuku-ku, 162-8655 Tokyo, Japan. ${ }^{4}$ Department of Pathology, Research Hospital, Institute of Medical Science, The University of Tokyo, 4-6-1 Shirokanedai, Minato-ku, 108-8639 Tokyo, Japan. ${ }^{5}$ Department of Applied Genomics, Research Hospital, Institute of Medical Science, The University of Tokyo, 4-6-1 Shirokanedai, Minato-ku, 108-8639 Tokyo, Japan. ${ }^{6}$ Division of Clinical Genome Research, Advanced Clinical Research Center, The Institute of Medical Science, The University of Tokyo, 4-6-1 Shirokanedai, Minato-ku, 108-8639 Tokyo, Japan.

Received: 13 August 2016 Accepted: 24 November 2016 Published online: 09 December 2016

\section{References}

1. Smeenk RM, van Velthuysen ML, Verwaal VJ, Zoetmulder FA. Appendiceal neoplasms and pseudomyxoma peritonei: a population based study. Eur J Surg Oncol. 2008;34:196-201.

2. Carr NJ, Finch J, llesley IC, Chandrakumaran K, Mohamed F, Mirnezami A, et al. Pathology and prognosis in pseudomyxoma peritonei: a review of 274 cases. J Clin Pathol. 2012;65:919-23.

3. Ronnett BM, Seidman JD. Mucinous tumors arising in ovarian mature cystic teratomas: relationship to the clinical syndrome of pseudomyxoma peritonei. Am J Surg Pathol. 2003;27:650-7.

4. McCarthy $\mathrm{JH}$, Aga R. A fallopian tube lesion of borderline malignancy associated with pseudo-myxoma peritonei. Histopathology. 1988;13:223-5.

5. Liu $Y$, Ishibashi H, Hirano M, Takeshita K, Mizumoto A, Ichinose M, et al. Cytoreductive surgery plus hyperthermic intraperitoneal chemotherapy for pseudomyxoma peritonei arising from urachus. Ann Surg Oncol. 2015;22:2799-805.

6. Goldstein PJ, Cabanas J, da Silva RG, Sugarbaker PH. Pseudomyxoma peritonei arising from colonic polyps. Eur J Surg Oncol. 2006;32:764-6. 
7. Giang TH, Ngoc TT, Hassell LA. Carcinoma involving the gallbladder: a retrospective review of 23 cases - pitfalls in diagnosis of gallbladder carcinoma. Diagn Pathol. 2012;7:10.

8. Ikejiri K, Anai H, Kitamura K, Yakabe S, Saku M, Yoshida K. Pseudomyxoma peritonei concomitant with early gastric cancer: report of a case. Surg Today. 1996:26:923-5.

9. Chejfec G, Rieker WJ, Jablokow VR, Gould VE. Pseudomyxoma peritonei associated with colloid carcinoma of the pancreas. Gastroenterology. 1986;90:202-5.

10. Goldin M, Li J, Amirrezvani A, Riker D. Pulmonary giant cell carcinoma associated with pseudomyxoma peritonei. J Bronchology Interv Pulmonol. 2012;19:50-3.

11. Hawes D, Robinson R, Wira R. Pseudomyxoma peritonei from metastatic colloid carcinoma of the breast. Gastrointest Radiol. 1991;16:80-2.

12. Bradley RF, Stewart JH, Russell GB, Levine EA, Geisinger KR. Pseudomyxoma peritonei of appendiceal origin: a clinicopathologic analysis of 101 patients uniformly treated at a single institution, with literature review. Am J Surg Pathol. 2006;30:551-9.

13. Shetty S, Thomas P, Ramanan B, Sharma P, Govindarajan V, Loggie B. Kras mutations and p53 overexpression in pseudomyxoma peritonei: association with phenotype and prognosis. J Surg Res. 2013;180:97-103.

14. Nishikawa G, Sekine S, Ogawa R, Matsubara A, Mori T, Taniguchi H, et al. Frequent GNAS mutations in low-grade appendiceal mucinous neoplasms. Br J Cancer. 2013;108:951-8.

15. Nummela $P$, Saarinen $L$, Thiel $A$, Jarvinen $P$, Lehtonen $R$, Lepisto $A$, et al. Genomic profile of pseudomyxoma peritonei analyzed using next-generation sequencing and immunohistochemistry. Int J Cancer. 2015;136:E282-9.

16. Noguchi R, Yano H, Gohda Y, Suda R, Igari T, Ohta Y, et al. Molecular profiles of high-grade and low-grade pseudomyxoma peritonei. Cancer Med. 2015;4:1809-16.

17. Pietrantonio F, Perrone F, Mennitto A, Gleeson EM, Milione M, Tamborini E, et al. Toward the molecular dissection of peritoneal pseudomyxoma. Ann Oncol. 2016;27:2097-103.

18. Pietrantonio F, Berenato R, Maggi C, Caporale M, Milione M, Perrone F, et al. GNAS mutations as prognostic biomarker in patients with relapsed peritoneal pseudomyxoma receiving metronomic capecitabine and bevacizumab: a clinical and translational study. J Transl Med. 2016;14:125.

19. Ansari N, Chandrakumaran K, Dayal S, Mohamed F, Cecil TD, Moran BJ. Cytoreductive surgery and hyperthermic intraperitoneal chemotherapy in 1000 patients with perforated appendiceal epithelial tumours. Eur J Surg Oncol. 2016;42:1035-41.

20. Lynch HT, de la Chapelle A. Hereditary colorectal cancer. N Engl J Med. 2003;348:919-32.

21. Mecklin JP. Frequency of hereditary colorectal carcinoma. Gastroenterology. 1987;93:1021-5.

22. Barrow E, Alduaij W, Robinson L, Shenton A, Clancy T, Lalloo F, et al. Colorectal cancer in HNPCC: cumulative lifetime incidence, survival and tumour distribution. A report of 121 families with proven mutations. Clin Genet. 2008;74:233-42.

23. van der Post RS, Kiemeney LA, Ligtenberg MJ, Witjes JA, Hulsbergen-van de Kaa CA, Bodmer D, et al. Risk of urothelial bladder cancer in Lynch syndrome is increased, in particular among MSH2 mutation carriers. J Med Genet. 2010:47:464-70.

24. Watson P, Vasen HF, Mecklin JP, Bernstein I, Aarnio M, Jarvinen HJ, et al. The risk of extra-colonic, extra-endometrial cancer in the lynch syndrome. Int J Cancer. 2008;123:444-9.

25. Hwang JH, So KA, Modi G, Lee JK, Lee NW, Lee KW, et al. Borderline-like mucinous tumor arising in mature cystic teratoma of the ovary associated with pseudomyxoma peritonei. Int J Gynecol Pathol. 2009:28:376-80.

26. Beck RP, Latour JP. Review of 1019 benign ovarian neoplasms. Obstet Gynecol. 1960;16:479-82.

27. Cariker M, Dockerty M. Mucinous cystadenomas and mucinous cystadenocarcinomas of the ovary; a clinical and pathological study of 355 cases. Cancer. 1954;7:302-10.

28. Okada S, Ohaki Y, Ogura J, Ishihara M, Kawamura T, Kumazaki T. Computed tomography and magnetic resonance imaging findings in cases of dermoid cyst coexisting with surface epithelial tumors in the same ovary. J Comput Assist Tomogr. 2004;28:169-73.

29. Le DT, Uram JN, Wang H, Bartlett BR, Kemberling H, Eyring AD, et al. PD-1 blockade in tumors with mismatch-repair deficiency. N Engl J Med. 2015;372:2509-20.
30. Chen CH, Huang RL, Yu MS, Wong $\sqcup$, Chao TF, Chu TY. Hereditary nonpolyposis colorectal cancer with gynecologic malignancies: report of two families in Taiwan. J Formosan Med Assoc (Taiwan yi zhi). 2001;100:269-73.

31. Smith J, Crowe K, McGaughran J, Robertson T. Sebaceous adenoma arising within an ovarian mature cystic teratoma in Muir-Torre syndrome. Ann Diagn Pathol. 2012;16:485-8.

\section{Submit your next manuscript to BioMed Central and we will help you at every step:}

- We accept pre-submission inquiries

- Our selector tool helps you to find the most relevant journal

- We provide round the clock customer support

- Convenient online submission

- Thorough peer review

- Inclusion in PubMed and all major indexing services

- Maximum visibility for your research

Submit your manuscript at www biomedcentral com/submit
Biomed Central 